\title{
Interleucina-4 no fluido gengival e no soro de pacientes com doença inflamatória intestinal e periodontite
}

\author{
Interleukin-4 in gingival crevicular fluid and serum of patients with inflammatory bowel disease and \\ periodontitis
}

Roberta Pedreira ${ }^{1}$, Juliana Menegat ${ }^{2}$, Fernanda Brito ${ }^{3}$, Ricardo G Fischer ${ }^{4}$, Carlos Marcelo S Figueredo ${ }^{5}$ ${ }^{1}$ Mestre em Periodontia - UERJ; ${ }^{2}$ Doutoranda em Periodontia - UERJ; ${ }^{3}$ Doutora em Periodontia - UERJ;

${ }^{4}$ Professor Titular de Periodontia - UERJ; ${ }^{5}$ Professor Adjunto de Periodontia - UERJ

\section{Resumo}

O objetivo foi avaliar os níveis de IL-4 no soro e fluido gengival (FG) de sítios com doença periodontal de pacientes com doença intestinal inflamatória (DII) e compará-los aos níveis encontrados em indivíduos sistemicamente saudáveis. Quarenta e cinco indivíduos com doença de Crohn (DC) $n=15$, Retocolite Ulcerativa Idiopática (RCUI) $n=15$ e grupo controle $(\mathrm{GC}) \mathrm{n}=15$. Profundidade de bolsa $(\mathrm{PB})$, nível de inserção anatômico $(\mathrm{NI})$, presença de placa, sangramento à sondagem foram avaliados em seis sítios por dente. Em cada paciente, o fluido de 4 sítios com periodontite ( $P B \geq 5 \mathrm{~mm}$ e $\mathrm{NI} \geq 3 \mathrm{~mm}$ ) e de 4 sítios com gengivite ( $P B \leq 3 \mathrm{~mm}$ e $\mathrm{NI} \leq 1 \mathrm{~mm}$ ) foram coletados. Quanto aos parâmetros clínicos periodontais avaliados em todos os dentes houve diferença na profundidade de bolsa entre os pacientes com DII e o controle, e para os dentes selecionados para coleta houve diferença na profundidade de bolsa entre os grupos de DC e GC nos sítios de gengivite. Nos sítios com gengivite, a quantidade total de IL-4 foi significativamente menor no grupo RCUI do que no grupo GC $(p=0.016)$. Nos sítios com periodontite, a quantidade total de IL-4 foi significativamente menor no grupo DC do que no grupo GC ( $p=0.029)$. Concluindo, os níveis da IL-4 estavam mais baixos nos sítios com periodontite dos pacientes com DII quando comparados aos controles, por outro lado não houve diferença na expressão no soro, indicando que uma possível baixa dessa citocina no fluido gengival pode estar relacionada com a patogenia da periodontite nesses pacientes.

Palavras-chave: Periodontite. Doenças Inflamatórias Intestinais. Interleucina-4.

\section{Abstract}

Our aim was to evaluate IL-4 levels in serum and gingival crevicular fluid (GCF) of periodontal disease sites in Inflammatory Bowel Disease (DII) patients and compared to systemic healthy controls. Forty-five individuals were allocated in three groups: Crohn disease (DC) $n=15$, Idiopathic Ulcerative Colitis (RCUI) $n=15$, and healthy group (GC) $n=15$. Probing depth (PB), attachment levels ( $\mathrm{NI}$ ), plaque presence, and blood on probing was examined in six sites per teeth. In every subject, GCF from 4 gingivitis sites (PPD $\leq 3 \mathrm{~mm}$ and $\mathrm{CAL} \leq 1 \mathrm{~mm}$ ) and from 4 periodontitis sites (PPD $\geq 5 \mathrm{~mm}$ and CAL $\geq 3 \mathrm{~mm}$ ) was collected with filter strips. RCUI presented higher number of former smokers than GC. There was difference in probe depth between DII group and control. When gingival crevicular fluid teeth selected were analyzed, the difference was shown only between DC and GC in gingivitis sites. In gingivitis sites, the total amount of IL-4 was significantly lower in the UC group than in the CG group ( $p=0.016)$. In periodontitis sites, the total amount of IL-4 was significantly lower in CD group than in the CG group $(p=0.029)$. The total amount of IL-4 was lower in UC group than in CD group ( $p=0.077)$. Similarly, IL-4 concentrations in both CD ( $p=0.096)$ and UC $(p=0.064)$ groups were lower than in CG. In conclusion, IBD patients had lower IL-4 levels in the GCF than healthy controls. Serum IL-4 levels showed no difference for all groups. Furthermore, IL-4 levels were lower in periodontitis sites from inflammatory bowel disease subjects; however, changes in IL-4 levels were not presented in serum levels, indicating IL-4 lower levels could be related to periodontal pathogenesis in these individuals. Keywords: Periodontitis. Inflammatory Bowel Diseases. Interleukin-4.

\section{INTRODUÇÃO}

A periodontite é uma doença multifatorial que resulta da interação entre as bactérias que colonizam a superfície radicular e os mecanismos de defesa do hospedeiro (GEMMELL et al., 1997; PAGE et al., 1997). Embora a prevalência de patógenos periodontais na população em geral seja moderada à alta, é reconhecido que os indivíduos não são igualmente suscetíveis ao desenvolvimento da doença (LINDHE et al.,1975; LOE et al., 1986; LIE et al., 1998). Isto é, a presença bacteriana Recebido em 26/02/2012; revisado em 27/03/2012.

Correspondência / Correspondence: Carlos Marcelo da S. Figueredo. Faculdade de Odontologia Universidade do Estado do Rio de Janeiro. Av. Boulevard 28 de setembro, 157 - Vila Isabel. CEP:20551-530 - RJ Tel.:(21)25876382 / (21) 99466114. E-mail: Roberta Pedreira- robertarp@bol.com.br; Carlos Marcelo da S. Figueredo - cmfigueredo@hotmail.com é fundamental para indução da resposta inflamatória nos tecidos periodontais, mas sua presença por si só, não é suficiente para destruição tecidual (PAGE et al., 1997).

Vários estudos têm sido realizados com objetivo de esclarecer as causas e mecanismos que podem resultar nesta suscetibilidade aumentada em alguns indivíduos (KINANE; BARTOLD, 2007). Entre os possíveis mecanismos estudados estão os relacionados à genética (BAKER, 2005) e às alterações quantitativas de citocinas no fluido gengival (HONDA et al., 2006). O equilíbrio entre citocinas pró e anti-inflamatórias parece ser importante para o estabelecimento e progressão da doença periodontal. Cochran (2008) mostra uma relação positiva entre a quantidade aumentada de citocinas próinflamatórias e a destruição periodontal, enquanto que 
Giannopoulou e colaboradores (2003) mostram que o dano periodontal pode estar relacionado à redução das citocinas anti-inflamatórias, entre elas a interleucina 4 (IL-4). (PRADEEP et al., 2009) demonstraram que a IL-4 apresenta-se diminuída no fluido gengival de indivíduos com periodontite e seu aumento tem sido relacionado com a remissão da doença periodontal.

A IL-4 é uma citocina anti-inflamatória que apresenta a capacidade de diminuir a produção de IL-1, fator de necrose tumoral á (TNFá) e IL-6 em monócitos do sangue periférico de humanos (ESSNER et al., 1989; DONNELLY et al., 1990). Uma menor quantidade de células T secretoras de IL-4 menor nas biópsias de crianças com DII (KARTUNNEN et al., 1994) e uma quantidade de RNA mensageiro de IL-4 transcrito menos detectável em indivíduos com DII (NIESSNER et al., 1995) Tal fato tem chamado a atenção para um possível papel da IL-4 na patogenia da doença periodontal nestes pacientes.

A doença inflamatória intestinal é dividida em duas entidades principais: Doença de Crohn (DC) e Retocolite Ulcerativa Idiopática (RCUI). Os mecanismos imunes da Retocolite Ulcerativa Idiopática e da doença de Crohn parecem ser distintos. Geralmente a doença de Crohn é considerada um processo inflamatório de resposta Th1 com grande expressão de interferon gama (INFã) e IL-2 que irá induzir a expressão da citocina IL12. Já a colite ulcerativa é atípica, há grande expressão de IL-5 e IL-13, citocinas comumente associadas à resposta Th2, entretanto a citocina IL-4 tipicamente Th2, não aparece aumentada, e há grande concentração de INFã. (BROWN; MAYER, 2007).

Como o trabalho recente realizado por nosso grupo mostra que a prevalência da periodontite está aumentada entre os indivíduos com DII (BRITO et al, 2008), o objetivo desta dissertação foi avaliar os níveis de IL-4 no fluido gengival (FG) de sítios com doença periodontal de pacientes com doença inflamatória intestinal e compará-los aos níveis encontrados em indivíduos sistemicamente saudáveis. Como objetivo secundário, avaliamos os níveis da IL-4 no soro desses pacientes.

\section{MATERIAIS E MÉTODOS}

Quinze pacientes com DC 38.2 (DP \pm 11.5 ) e 15 pacientes com RCUI portadores de periodontite crônica 43.2 ( $D P \pm 7.2$ ) foram selecionados de um estudo previamente realizado sobre a prevalência de periodontite em pacientes com DII (BRITO et al., 2008). Os pacientes eram acompanhados nos ambulatórios de DII do Hospital Universitário Pedro Ernesto/ Universidade do Estado do Rio de Janeiro (HUPE-UERJ) e do Hospital Universitário Clementino Fraga Filho/ Universidade Federal do Rio de Janeiro (HUCFF-UFRJ), ambos localizados na cidade do Rio de Janeiro, Brasil. O grupo controle foi constituído por 15 indivíduos 45.0
(DP \pm 10.5 ) sem sinais de doenças sistêmicas, que tinham periodontite crônica generalizada e haviam participado do mesmo estudo de prevalência (BRITO et al., 2008).

Foram considerados como critério de inclusão: ter participado do estudo prévio sobre a prevalência de periodontite em pacientes com DII (BRITO et al., 2008) e possuir, pelo menos, quatro sítios com periodontite e 4 sítios com gengivite em dentes diferentes com perda de inserção anatômica $\geq 5 \mathrm{~mm}$. Foram considerados como critérios de exclusão: gravidez, tratamento periodontal prévio à coleta e uso de antibióticos há menos de dois meses da realização da coleta do fluido gengival. Este estudo foi aprovado pelos Comitês de Ética em Pesquisa da UERJ e HUCFF. Os pacientes assinaram um Termo de Consentimento antes de serem examinados.

Os integrantes de cada grupo foram entrevistados através de um questionário no qual constavam os seguintes dados: sexo, etnia (branco, pardo ou negro), idade, hábito de fumar (fumante, não fumante ou exfumante), duração do habito de fumar, a quantidade de cigarros por dia, tempo da doença inflamatória intestinal, história familiar, localização da doença, tipo da doença, presença de manifestações extra-intestinais, medicamentos utilizados específicos ou não para a DII, resposta ao tratamento com esteróides, história prévia de doença cardiovascular (hipertensão, coronariopatias), tratamento periodontal anterior. Os dados obtidos no questionário foram posteriormente confirmados com os dados obtidos dos prontuários médicos. A atividade de doença foi avaliada clinicamente e laboratorialmente empregando-se o índice de atividade da doença de Crohn (CDAI) Best (1976) e o Truelove e colaboradores (1955) para a RCUI.

Os pacientes do grupo DC utilizavam como medicação: imunomoduladores $(n=6)$, derivados do ácido 5 aminosalicilato $(n=2)$, corticóides $(n=4)$, imunomoduladores + derivados do ácido 5 aminosalicilato $(n=1)$. Dois pacientes do grupo $D C$ não tomavam nenhuma medicação. Os pacientes do grupo RCUI utilizavam como medicação: derivados do ácido 5 aminosalicilato $(n=8)$, imunomoduladores $(n=1)$, corticóides $(n=2)$ e imunomoduladores + derivados do ácido 5 aminosalicilato $(n=4)$.

Todos os dentes presentes, com exceção dos terceiros molares, foram examinados. As mensurações foram realizadas em seis sítios por dente (distovestibular, vestibular, mesio-vestibular, disto-lingual, lingual e mesio-lingual). Os parâmetros clínicos avaliados foram: (1) profundidade de bolsa (PB), distância entre a margem gengival e a porção mais apical sondável, (2) nível de inserção anatômica (NI), distância entre a junção amelo-cementária e a porção mais apical sondável, (3) presença de placa e (4) presença de sangramento a sondagem (SS).

A sonda utilizada para realização de todas as medidas foi uma sonda periodontal milimetrada (PCR 
15, Hu-Friedy ${ }^{\bullet}$, Chicago, IL, EUA). O exame clínico e a coleta das amostras foram realizados por duas examinadoras previamente calibradas ( $K=0.775$, $\mathrm{p}<0.001)$.

Foi coletado aproximadamente $8 \mathrm{~mL}$ de sangue de cada paciente em um tubo tijolo (Vacutainer ${ }^{\bullet}$, Juiz de Fora, Brasil) de $10 \mathrm{ml}$, a vácuo com gel, pelas enfermeiras do HUPE ou do HUCFF. Após a coleta, o material foi centrifugado por 5 minutos. O soro foi congelado a 80 ㄷ C até o momento da análise que foi realizada no laboratório do Departamento de Periodontia do Karolinska Institutet (Estocolmo, Suécia). Em um paciente do grupo DC o soro não foi coletado.

Em cada paciente, o fluido de sítios inflamados, quatro sítios com periodontite (PBS $\geq 5 \mathrm{~mm}$ e $\mathrm{NI} \geq 3 \mathrm{~mm}$ ) e 4 sítios com gengivite ( $P B$ e $\mathrm{NI} \leq 3 \mathrm{~mm}$ ) foram analisados. Os sítios selecionados para análise foram isolados com rolos de algodão e, quando necessário, a placa supragengival foi removida. Os sítios foram secados levemente com uma seringa de ar. O fluido gengival foi coletado através de pontas de papel absorventes pré-fabricadas (Proflow, Inc., Amityville, NY, EUA). A ponta de papel era introduzida na bolsa até que uma leve resistência fosse sentida e permaneceu no local da coleta durante trinta segundos. As amostras contendo sangue foram descartadas. O volume absorvido em cada ponta de papel foi determinado pelo Periotron $8000^{\circ}$ (IDE, Interstate, Amityville, NY, EUA) previamente calibrado. As leituras do Periotron foram convertidas em volume, utilizando-se como referência a curva padrão obtida durante a calibragem do aparelho. Em cada pessoa, as pontas de papel provenientes da mesma categoria de análise (periodontite ou gengivite) foram agrupadas em um frasco tipo Eppendorf e diluídas em $1 \mathrm{ml}$ de solução salina fosfato-tamponada (PBS). Após diluição por 40 minutos em temperatura ambiente, as pontas de papel foram removidas e as amostras foram imediatamente centrifugadas a $3000 \mathrm{rpm}$ por 5 minutos. O sobrenadante foi coletado e congelado a -80 ㄷ C até o momento da análise. A análise dos dados foi realizada no laboratório do departamento de periodontia do Karolinska Institutet (Estocolmo, Suécia). A análise com o LUMINEX ${ }^{\circledR}$ (xMAP technology, Luminex, Austin, Texas, EUA) foi utilizada para mensuração de IL-4. Cinquenta $\mu \mathrm{L}$ de fluido gengival e $50 \mu \mathrm{L}$ de soro foram analisados utilizando um kit comercial Lincoplex ${ }^{\circledR}$ (Millipore, Missouri, USA), de acordo com as recomendações do fabricante. Os resultados foram calculados pelo programa Bio-Plex Manager Software (Bio-Rad Laboratories, Hercules, CA, EUA).

\section{ANÁLISE ESTATÍSTICA}

Valores distribuídos normalmente foram apresentados através de média e desvio-padrão enquanto que valores não distribuídos normalmente foram apresentados como mediana e intervalo interquartil. O teste $T$ foi utilizado para a comparação de dados paramétricos. O teste Mann-Whitney $U$ foi utilizado para comparar os dados não-paramétricos entre os grupos. O teste de Wilcoxon foi utilizado para comparar dados não-paramétricos entre os sítios com gengivite e periodontite no mesmo grupo. O coeficiente de correlação de Pearson foi utilizado na análise da correlação entre as citocinas no soro e no fluido. 0 nível de significância foi estabelecido em $5 \%$ ( $p<0.05)$. As análises foram realizadas no programa Statistica, versão 7.1, 2005 (STATSOFT, 2005).

\section{RESULTADOS}

\section{Características clínicas e periodontais}

As características clínicas estão apresentadas na tabela 1. Os grupos estão distribuídos sem diferença estatisticamente significante em relação à idade, distribuição entre sexo, raça, fumantes, não fumantes, diabetes e hipertensão. Os pacientes eram considerados não fumantes, quando relatavam nunca ter fumado, fumante se fumasse independente da quantidade de cigarros/dia e da frequência, e ex-fumante se tivesse cessado o hábito de fumar. Entre os grupos com doença inflamatória intestinal não há diferença estatisticamente significante para história familiar de doença inflamatória intestinal, tempo de diagnóstico da DII, manifestação extra-intestinais, doença ativa. Entre o grupo de RCUI e o grupo controle (GC) houve diferença estatisticamente significante para a quantidade de exfumantes, sendo que no grupo de RCUI o número de exfumantes era maior que no grupo controle.

Na tabela 2 são apresentados os dados sobre os parâmetros clínicos dos sítios de gengivite submetidos à coleta. Para o parâmetro de profundidade de bolsa, nos sítios com gengivite nos pacientes com periodontite, houve diferença estatisticamente significante entre os indivíduos com DC e os indivíduos do GC. Entre o grupo controle e os indivíduos com RCUI a diferença encontrada não foi estatisticamente significante. Não houve diferença os entre os grupos dos indivíduos com RCUI e DC. Não houve diferença entre os grupos para os parâmetros clínicos de nível de inserção, percentual de sítios com presença de placa. Nos sítios submetidos à coleta não houve diferença na média do volume de fluido entre os três grupos.

Quando analisados os sítios com periodontite, não houve diferença entre os grupos para os parâmetros clínicos de profundidade de bolsa, nível de inserção e percentual de presença de placa bacteriana. Os dados clínicos dos sítios com periodontite submetidos à coleta foram apresentados na tabela 3 .

\section{Análise do fluido gengival e do soro}

Os dados dos níveis de IL-4 no fluido gengival dos sítios com gengivite nos indivíduos com periodontite dos grupos com DC, RCUI e GC são apresentados na tabela 4. Os dados dos níveis de IL-4 no fluido gengival 
Tabela 1 - Características clínicas apresentadas em número de indivíduos e porcentagem. A idade é expressa em média e desvio-padrão (teste T). O tempo de diagnóstico de doença é expresso em mediana e variação interquartil

(teste de Mann-Whitney U). Para todas as outras variáveis o teste $\div^{2}$ foi utilizado. Foram avaliados 15 pacientes com doença de chron (DC), 15 pacientes com Retocolite Ulcerativa idiopática (RCUI) e 15 pacientes do Grupo controle (GC). P1 significância da diferença entre os grupos DC e GC, p2 significância da diferença entre os grupos RCUI e GC, p3 significância da diferenca entre os grupos DC e RCUI. DIl doenca inflamatória intestinal.

\begin{tabular}{l|llllll} 
& DC & $p 1$ & GC & $p 2$ & RCUI & $p$ 3 \\
\hline Idade & $38.2(11.5)$ & 0.085 & $43.3(7.2)$ & 0.157 & $45.0(10.5)$ & 0.749 \\
Homens & $8(53.3 \%)$ & 0.715 & $7(46.7 \%)$ & 1.000 & $7(46.7 \%)$ & 0.715 \\
Mulheres & $7(46.7 \%)$ & 0.715 & $8(53.3 \%)$ & 1.000 & $8(53.3 \%)$ & 0.715 \\
Brancos & $9(60.0 \%)$ & 0.273 & $7(46.7 \%)$ & 0.712 & $6(40.0 \%)$ & 0.273 \\
Pardos & $6(40.0 \%)$ & 1.000 & $6(40.0 \%)$ & 0.464 & $8(53.3 \%)$ & 0.464 \\
Negros & 0 & 0.143 & $2(13.3 \%)$ & 0.542 & $1(6.7 \%)$ & 0.309 \\
Fumantes & $3(20.0 \%)$ & 0.624 & $2(13.3 \%)$ & 0.542 & $1(6.7 \%)$ & 0.282 \\
Não Fumantes & $8(53.3 \%)$ & 0.255 & $11(73.3 \%)$ & 0.136 & $7(46.7 \%)$ & 0.715 \\
Ex-fumantes & $4(26.7 \%)$ & 0.361 & $2(13.3 \%)$ & 0.046 & $7(46.7 \%)$ & 0.255 \\
Hipertensos & $3(20.0 \%)$ & 0.067 & 0 & 0.067 & $3(20.0 \%)$ & 1.0 \\
Diabéticos & 0 & 0.309 & 0 & 0.309 & $1(6.7 \%)$ & 0.309 \\
Doença Ativa & $5(33.3 \%)$ & & & & $3(20.0 \%)$ & 0.409 \\
\hline
\end{tabular}

Tabela 2 - Parâmetros clínicos nos sítios com gengivite dos indivíduos com periodontite. Os dados são apresentados em mediana e variação interquartil (teste de Mann-Whitney U). O volume é expresso em média e desvio-padrão. Os valores das citocinas estão expressos em picograma. P1 significância da diferença entre os grupos DC (Doença de Crohn) e GC (grupo controle) , p2 significância da diferença entre os grupos RCUI (Retocolite ulcerativa idiopática) e GC, p3 significância da diferença entre os grupos DC e RCUI, PB profundidade de bolsa, NI nível de inserção

\begin{tabular}{l|lccccc}
\hline & \multicolumn{1}{|c}{$\begin{array}{c}\text { DC } \\
(n=15)\end{array}$} & \multicolumn{1}{c}{$p$} & $\begin{array}{c}\text { GC } \\
(n=15)\end{array}$ & $p 2$ & $\begin{array}{c}R C U I \\
(n=15)\end{array}$ & $p 3$ \\
\hline PB (mm) & $2.7(0.5)$ & 0.036 & $3.0(0.4)$ & 0.056 & $2.5(0.8)$ & 0.597 \\
NI (mm) & $0.7(0.5)$ & 0.072 & $1.0(0.2)$ & 0.469 & $1.0(0.5)$ & 0.486 \\
\% sítios com placa & $50.0(100.0)$ & 0.078 & $100.0(50.0)$ & 0.469 & $1.0(0.5)$ & 0.594 \\
Volume & $1.9(0.9)$ & 0.785 & $1.8(0.8)$ & 0.921 & $1.8(1.2)$ & 0.758 \\
IL-4 total & $0.7(1.5)$ & 0.256 & $1.2(2.1)$ & 0.016 & $0(0.8)$ & 0.136 \\
IL-4 (conc) & $0.2(1.0)$ & 0.214 & $0.9(1.7)$ & 0.059 & $0(0.5)$ & 0.293 \\
\hline
\end{tabular}

Tabela 3 - Parâmetros clínicos nos sítios com periodontite. Os dados são apresentados em mediana e variação interquartil (teste de Mann-Whitney U). O volume é expresso em média e desvio-padrão. Os valores das citocinas estão expressos em picograma. p1 significância da diferença entre os grupos DC (Doença de Crohn) e GC (Grupo controle), p2 significância da diferença entre os grupos RCUI (Retocolite ulcerativa idiopática) e GC, p3 significância da diferença entre os grupos DC e RCUI, PB profundidade de bolsa, NI nível de inserção.

\begin{tabular}{l|llllll}
\hline & $\begin{array}{l}D C \\
(n=15)\end{array}$ & $p 1$ & $\begin{array}{l}G C \\
(n=15)\end{array}$ & $p 2$ & $\begin{array}{l}R C U I \\
(n=15)\end{array}$ & $p 3$ \\
\hline PB (mm) & $5.2(1.5)$ & 0.517 & $5.0(0.8)$ & 0.664 & $5.2(1.3)$ & 0.754 \\
NI (mm) & $3.7(2.7)$ & 0.466 & $3.0(1.2)$ & 0.288 & $4.0(1.2)$ & 0.867 \\
\% sítios com placa & $66.7(75.0)$ & 0.698 & $75.0(75.0)$ & 0.347 & $60.0(80.0)$ & 0.627 \\
Volume & $2.9(1.0)$ & 0.545 & $2.6(1.1)$ & 0.388 & $3.0(1.5)$ & 0.684 \\
IL-4 total & $0.9(1.0)$ & 0.029 & $1.9(2.6)$ & 0.077 & $1.0(1.7)$ & 0.819 \\
IL-4 (conc) & $0.4(0.5)$ & 0.096 & $0.7(1.6)$ & 0.064 & $0.3(0.5)$ & 0.917 \\
\hline
\end{tabular}

dos sítios com periodontite dos grupos com DC, RCUI e GC são apresentados na tabela 5, na qual mostra que há uma diferença estatisticamente significante entre os pacientes com DC e GC para quantidade total de IL-4. Mas esta diferença não é observada entre os grupos com DII e o grupo controle. 
Os dados relacionados à comparação do nível de IL-4 no fluido gengival dos sítios com gengivite e periodontite nos pacientes com DC, RCUI e GC estão apresentados na tabela 6. Houve diferença estatisticamente significante entre a quantidade total de IL-4 no fluido gengival dos sítios de gengivite e periodontite tanto no grupo controle e no grupo RCUI. Mas esta diferença não foi observada no grupo DC. Para concentração de IL-4 no fluido gengival não houve diferença entre os sítios com gengivite e periodontite em todos os grupos.

A concentração de IL-4 no soro dos pacientes incluídos no estudo, apresentada em média e intervalo interquartil, foi de 0 (1.6), 0 (10.7), 0 (10.6) para os grupos DC, RCUI e GC, respectivamente. Não houve diferença estatisticamente significante entre os grupos, sendo de DC e GC ( $p=0.769)$, RCUI e GC ( $p=0.548)$ e DC e RCUI $(p=0.625)$. No grupo DC um paciente não foi submetido à coleta de soro.

\section{DISCUSSÃO}

Observamos uma menor quantidade de IL-4 no fluido gengival dos indivíduos com DII comparada com o grupo GC. Quando avaliada a concentração de IL-4 não há diferença entre os grupos. Quanto à discrepância entre quantidade total e concentração, esta pode estar relacionada à diluição da citocina no volume do fluido que normalmente encontra-se aumentado nos sítios com inflamação como demonstrado por Gamonal e colaboradores (2003) e Ikezawa e colaboradores (2005). Não existem estudos que avaliam os níveis de IL-4 no fluido gengival de pacientes com DII. No entanto, já foi mostrado que a IL4 tem relação com a indução de apoptose em macrófagos e que seus baixos níveis nos sítios com periodontite, podem levar ao aumento local de macrófagos ativados e consequentemente ao aumento na destruição do periodonto (YAMAMOTO et al., 1996). Sendo até sugerido que a ausência de IL-4 estaria relacionada à progressão da gengivite para periodontite (SHAPIRA et al., 1992). Este fato pode está relacionado ao perfil do paciente com DII, visto que uma quantidade diminuída de células T secretoras de IL-4 foi mostrada em biópsias intestinais de crianças com DII (KARTTUNNEN et al., 1994). E também pode ter relação com a prevalência de periodontite aumentada nos indivíduos com DII, como demonstrada por Brito e colaboradores. (2008).

Nosso estudo mostra que os sítios com periodontite apresentavam níveis maiores de IL-4 quando comparados aos sítios com gengivite nos grupos RCUI e GC. Estudos realizados em indivíduos sistemicamente saudáveis mostram uma maior quantidade de IL-4 em pacientes com gengivite em comparação com periodontite (TSAI ET AL., 2007; PRADEEP, et al., 2009). A razão para essa diferença não está clara, porém isto pode estar relacionado ao fato de compararmos níveis de IL-4 em sítios de gengivite e periodontite nos indivíduos com periodontite. Para podermos comparar esses dados com a literatura, seria necessário um grupo de indivíduos somente com gengivite. Todavia, utilizando uma metodologia diferente do estudo que realizamos, (BICKEL et al., 2001) encontraram uma maior concentração nas células expressando o RNAm de IL-4, em biópsias de tecido gengival de lesões periodontais classificadas como progressivas, o que pode justificar a maior presença de IL-4 no fluido dos sítios com periodontite.

Entre os indivíduos incluídos no estudo, alguns eram fumantes. No entanto, não houve diferença estatística para quantidade de fumantes entre os grupos selecionados para coleta de fluido gengival. O fumo é reconhecidamente um fator de risco para periodontite (RIVERA-HIDALGO, 2003), que inclusive, tem a capacidade de aumentar o volume (USTUN; ALPTEKIN, 2007) e alterar os níveis de citocinas no fluido gengival em indivíduos periodontalmente saudáveis (KAMMA et al., 2004). Já em relação a DII, o efeito do fumo pode variar, resultando mais complicações, maior probabilidade de internação e necessidade de procedimentos cirúrgicos para indivíduos com Doença de Crohn. No entanto, este efeito não é observado nos indivíduos com DC restrita ao cólon, onde o cigarro parece ter um efeito positivo como o que ocorre na RCUI, onde o hábito de fumar parece reduzir a necessidade colectomia. Parar de fumar pode resultar na piora do paciente com RCUI e melhorar o estado do paciente com DC. (LAKATOS et al., 2007; REDDY; TREMAINE, 2008).

Os pacientes com DII fazem uso de medicações imunomoduladoras para o controle da doença. Os medicamentos variam de acordo com a extensão e grau de severidade da doença (BAUMGART, 2009). Com relação ao uso de corticóide, embora seja mostrada a relação entre a sua utilização e a osteoporose, esta não parece estar relacionada à perda de osso alveolar (BURKETT, 1972). Já foi demonstrado que o uso de corticóide não tem influência na frequência e nem na severidade da periodontite (SAFKAN; KNUUTILLA, 1984). No entanto, não foram encontrados estudos que mostrem a influência do uso de corticóide e dos outros medicamentos utilizados pelos pacientes incluídos no estudo, nos níveis de IL-4 no fluido gengival. Entretanto, em biópsias intestinais de pacientes com DII, onde o número de células T secretoras de IL-4 estava diminuído nos pacientes com DC, este fato não estava relacionado ao uso de corticóide (KARTTUNNEN et al., 1994).

Em relação à profundidade de bolsa, houve diferença estatisticamente significante entre os pacientes com DC e GC. Porém, consideramos que esta diferença entre os grupos não foi clinicamente relevante, visto que não houve diferença para o nível de inserção periodontal.

Entendemos que mais estudos com uma amostra maior, separação dos grupos por medicação, análise do fluido em pacientes periodontalmente sadios, com gengivite e o efeito do tratamento não cirúrgico nestes 
pacientes, são necessários e poderiam contribuir para uma melhor compreensão da patogênese da doença periodontal.

\section{CONCLUSÃO}

Concluindo, os níveis da IL-4 estavam mais baixos nos sítios com periodontite dos pacientes com doença inflamatória intestinal quando comparados aos controles, por outro lado, não houve diferença na expressão no soro, indicando que uma possível baixa dessa citocina no fluido gengival pode estar relacionada com a patogenia da periodontite nesses pacientes.

\section{REFERÊNCIAS}

BAKER, Pamela J. Genetic control of the immune response in pathogenesis. J. Periodontol., Indianapolis, v. 76, n.11 suppl, p.2042-6, Nov. 2005.

BAUMGART, Daniel. C. The diagnosis and treatment of Crohn's disease and ulcerative colitis. Dtsch. Arztebl. Int., Cologne, v. 106, n.8, p.123-33, Feb. 2009.

BEST, W. R. et al. Development of a Crohn's disease activity index. National Cooperative Crohn's Disease Study. Gastroenterology, Baltimore, v. 70, n.3, p.439-44, mar. 1976.

BURKETT, LW. Oral Medicine Diagnosis and Treatment. $6^{\text {th }}$ edn. Philadelphia, PA: J.B. Lippincott, 1972. p. 273-282.

BICKEL, M., B. Axtelius, et al. Cytokine gene expression in chronic periodontitis. J. Clin. Periodontol., Copenhagen, v. 28, n.9, p.8407, sep. 2001.

BRITO, F. et al. Prevalence of periodontitis and DMFT index in patients with Crohn's disease and ulcerative colitis. J. Clin. Periodontol., Copenhagen, v. 35, n. 6, p. 555-60, jun. 2008.

BROWN, Steven. J.; MAYER, Lloyd. The immune response in inflammatory bowel disease. Am. J. Gastroenterol., New York, v. 102, n. 9, p. 2058-69, sep. 2007.

HRAN, David L. Inflammation and bone loss in periodontal disease. J. Periodontol., Indianapolis, v. 79, n. 8 suppl, p. 156976, aug. 2008.

DONNELLY, R. P. et al. Differential regulation of IL-1 production in human monocytes by IFN-gamma and IL-4. J. Immunol., Baltimore, v. 145 , n. 2, p. 569-75. jul. 1990.

ESSNER, R. et al. IL-4 down-regulates IL-1 and TNF gene expression in human monocytes. J. Immunol., Rockville Pike, v. 142, n. 11, p. 3857-61, jul. 1989.

GAMONAL, J. et al. Delayed neutrophil apoptosis in chronic periodontitis patients. J. Clin. Periodontol., Copenhagen, v. 30, n. 7, p. 616-23, jul. 2003.

GEMMELL, E.; MARSHALL, R.I.; SEYMOUR, G.J. Cytokines and prostaglandins in immune homeostasis and tissue destruction in periodontal disease. Periodontol 2000, Copenhagen, v. 14, p. 112-43, jun. 1997.

GIANNOPOULOU, C.; KAMMA, J.J.; MOMBELLI, A. Effect of inflammation, smoking and stress on gingival crevicular fluid cytokine level. J. Clin. Periodontol., Copenhagen, v. 30, n. 2, p. 145-53, feb. 2003.

HONDA, T. et al. Balance of inflammatory response in stable gingivitis and progressive periodontitis lesions. Clin. Exp. Immunol., London, v. 144, n. 1, p. 35-40, Apr. 2006.

IKEZAWA, I. et al. Imbalance between soluble tumour necrosis factor receptors type 1 and 2 in chronic periodontitis. J. Clin. Periodontol., Copenhagen, v. 32, n.10, p.1047-54, oct. 2005.

KAMMA, J. J. et al. Cytokine profile in gingival crevicular fluid of aggressive periodontitis: influence of smoking and stress. J. Clin. Periodontol., Copenhagen, v. 31, n. 10, p. 894-902, oct. 2004

KARTTUNNEN, R. et al. Decreased mucosal interleukin-4 (IL-4) production in gut inflammation. J. Clin. Pathol., London, v. 47, n. 11, p. 1015-8, nov. 1994.

KINANE, Demis F. MARK BARTOLD, P. Clinical relevance of the host responses of periodontitis. Periodontol 2000, Copenhagen, v. 43, n. 1 , p. $278-93$, feb. 2007.

LAKATOS, P. L.; SZAMOSI, T.; LAKATOS, L. Smoking in inflammatory bowel diseases: good, bad or ugly? World J. Gastroenterol., Beijing, v. 13 , n. 46 , p. $6134-9$, dec. 2007

LIE, M. A. et al. Oral microbiota in smokers and non-smokers in natural and experimentally-induced gingivitis. J. Clin. Periodontol., Copenhagen, v. 25, n.8, p.677-686, aug. 1998.

LINDHE, J.; HAMP, S.E.; LOE, H. Plaque induced periodontal disease in beagle dogs. A 4-year clinical, roentgenographical and histometrical study. J. Periodontal. Res., Copenhagen, v. 10, n. 5, p. 243-55, nov.1975.

LOE, H. et al. Natural history of periodontal disease in man. Rapid, moderate and no loss of attachment in Sri Lankan laborers 14 to 46 years of age. J. Clin. Periodontol., Copenhagen, v. 13, n. 5, p. 431-45, may. 1986.

NIESSNER, M.; B. A. VOLK, B.A. Altered Th1/Th2 cytokine profiles in the intestinal mucosa of patients with inflammatory bowel disease as assessed by quantitative reversed transcribed polymerase chain reaction (RT-PCR). Clin. Exp. Immunol., London, v. 101, n. 3, p. 428-435, sep. 1995.

PAGE, R. C. et al. Advances in the pathogenesis of periodontitis: summary of developments, clinical implications and future directions. Periodontol 2000, Copenhagen, v. 14, p. 216-248, jun. 1997.

PRADEEP, A. R. et al. Gingival crevicular fluid and plasma levels of neuropeptide Substance-P in periodontal health, disease and after nonsurgical therapy. J. Periodontal. Res., Copenhagen, v. 44, n. 2 , p. $232-7$, apr. 2009

REDDY, Raghuram P.; TREMAINE, William J.. Is there a threshold for the deleterious effect of smoking in Crohn's disease? Inflamm. Bowel Dis., Hoboken, v. 14 n. 2, p.S16-17, oct. 2008.

RIVERA-HIDALGO, Francisco. Smoking and periodontal disease. Periodontol 2000, Copenhagen. v. 32, n. 1, p. 50-58, may. 2003. 
SAFKAN, B.; KNUUTTILA, Matti. Corticosteroid therapy and periodontal disease. J. Clin. Periodontol., Copenhagen, v. 11, n. 8, p. 515-22, sep. 1984.

SHAPIRA, L.; VAN DYKE, TE.; HART, TC.; . A localized absence of interleukin-4 triggers periodontal disease activity: a novel hypothesis. Med. Hypotheses., Edinburgh, v. 39, n. 4, p. 319-322, dec. 1992.

STATSOFT. Statistica (data analysis software system). Version 7.1. 2005. Available at: <www.statsoft.com>.

TRUELOVE, Sidney C.; WITTS, Leslie. Cortisone in ulcerative colitis; final report on a therapeutic trial. Br. Med. J., London, v. 2, n. 4947, p. 1041-1048, oct. 1955 .
TSAI, C. C. et al. Changes in gingival crevicular fluid interleukin-4 and interferon-gamma in patients with chronic periodontitis before and after periodontal initial therapy. Kaohsiung J. Med. Sci., Taiwan, v. 23, n.1, p.1-7, jan. 2007.

USTUN, Kemal; ALPTEKIN, Nilgun. The effect of tobacco smoking on gingival crevicular fluid volume. Eur. J. Dent., Gaziantep, v. 1, n. 4, p. 236-239, oct. 2007.

YAMAMOTO, M. et al. Absence of exogenous interleukin-4-induced apoptosis of gingival macrophages may contribute to chronic inflammation in periodontal diseases. Am. J. Pathol., New York, v. 148, n. 1, p. 331-339, jan. 1996 